University of Texas at El Paso

ScholarWorks@UTEP

$10-2001$

\title{
Aerospace Applications of Intervals: From Geospatial Data Processing to Fault Detection in Aerospace Structures
}

Vladik Kreinovich

The University of Texas at El Paso, vladik@utep.edu

Scott A. Starks

The University of Texas at El Paso, sstarks@utep.edu

Follow this and additional works at: https://scholarworks.utep.edu/cs_techrep

Part of the Computer Engineering Commons

Comments:

UTEP-CS-01-31.

Published in International Journal of Uncertainty, Fuzziness, and Knowledge Based Systems, 2001, Vol. 9, No. 6, pp. 721-730.

\section{Recommended Citation}

Kreinovich, Vladik and Starks, Scott A., "Aerospace Applications of Intervals: From Geospatial Data Processing to Fault Detection in Aerospace Structures" (2001). Departmental Technical Reports (CS). 418.

https://scholarworks.utep.edu/cs_techrep/418

This Article is brought to you for free and open access by the Computer Science at ScholarWorks@UTEP. It has been accepted for inclusion in Departmental Technical Reports (CS) by an authorized administrator of ScholarWorks@UTEP. For more information, please contact Iweber@utep.edu. 
International Journal of Uncertainty, Fuzziness and Knowledge-Based Systems

Vol. 0, No. 0 (1993) 000-000

(C) World Scientific Publishing Company

\title{
AEROSPACE APPLICATIONS OF INTERVALS: FROM GEOSPATIAL DATA PROCESSING TO FAULT DETECTION IN AEROSPACE STRUCTURES
}

\author{
VLADIK KREINOVICH and SCOTT A. STARKS \\ University of Texas at El Paso and NASA Pan-American Center for Earth \\ and Environmental Studies (PACES) \\ 500 West University Avenue, El Paso, TX 79968,USA, vladik@cs.utep.edu
}

Received March 2001

Revised October 2001

\begin{abstract}
This paper presents a brief introduction into interval computations and their use in aerospace applications.

Keywords: Interval computations; aerospace applications
\end{abstract}

\section{Introduction: Data Processing and Interval Computations}

\subsection{Data Processing}

In many real-life problems, we are interested in the value $y$ of a physical quantity which is difficult or impossible to measure directly. For example, we cannot directly measure the distance to a star, or the amount of oil in a given area. To measure this quantity $y$, we:

- measure some other quantities $x_{1}, \ldots, x_{n}$ which are related to $y$ by a known dependence $y=f\left(x_{1}, \ldots, x_{n}\right)$, and then

- compute the estimate $\widetilde{y}$ for the desired quantity $y$ by applying the algorithm $f$ to the results $\widetilde{x}_{i}$ of measuring the quantities $x_{i}: \widetilde{y}=f\left(\widetilde{x}_{1}, \ldots, \widetilde{x}_{n}\right)$.

This two-stage process is called indirect measurement, and computing $f$ is called data processing.

For example, to estimate the amount of oil in a given area, we may use geophysical data plus satellite images of this area.

\subsection{Error Estimation of the Results of Data Processing: Mathematical Statistics and Interval Computations}

Values $\widetilde{x}_{i}$ come from measurements, and measurements are never $100 \%$ accurate; therefore, $\widetilde{x}_{i} \neq x_{i}$. Due to the inaccuracies $\Delta x_{i}=\widetilde{x}_{i}-x_{i}$ of direct measurements, 
the result $\widetilde{y}=f\left(\widetilde{x}_{1}, \ldots, \widetilde{x}_{n}\right)$ is, in general, different from the desired value $y=$ $f\left(x_{1}, \ldots, x_{n}\right): \Delta y=\widetilde{y}-y \neq 0$. In practical applications, it is extremely important to know what are the possible values of the difference $\Delta y$.

For example, if our estimate for amount of oil in a given area is $\approx 100 \mathrm{mln}$. ton, then whether we start exploiting this oil or not depends on the accuracy of this estimate:

- If the measurement error $\Delta y$ does not exceed $10 \mathrm{mln}$. ton, then the actual value can be anywhere from 90 to 100, and we should recommend exploitation.

- On the other hand, if the measurement error $\Delta y$ can be as large as 100 mln. ton, then this means that the actual value $y$ can actually be equal to 0 (meaning that there may be no oil at all). In this case, further, more accurate measurements are needed because we can make a decision.

To estimate $\Delta y$, we must have some information about the errors $\Delta x_{i}$ of direct measurements. What type of information can we have?

- The manufacturer of the measuring instrument gives us a guaranteed error $\Delta_{i}$, i.e., a value for which $\left|\Delta x_{i}\right| \leq \Delta_{i}$. (Without such a guarantee, a measurement result does not restrict possible values of $x_{i}$ and thus, it is not a measurement.)

- In some cases, in addition to the upper bounds $\Delta_{i}$, we know probabilities of different values of $\Delta x_{i}$.

If we know probabilities, then we have a typical problem of mathematical statistics: given probability distributions for $\Delta x_{i}=\widetilde{x}_{i}-\Delta x_{i}$, find the probability distribution for $y=f\left(x_{1}, \ldots, x_{n}\right)$. To get the probabilities of $\Delta x_{i}$, we calibrate the measuring instrument, i.e., we compare its results with the results of a better (standard) measuring instrument.

However, there are two important situations when we do not know these probabilities:

- In fundamental physics, we perform measurements on the cutting edge, so no better instrument is possible at all.

- In manufacturing, calibration of all sensors is potentially possible, but, in practice, too expensive.

When we do not know the probabilities, we only know that $\left|\widetilde{x}_{i}-x_{i}\right| \leq \Delta_{i}$, i.e., the only information about $x_{i}$ is that $x_{i}$ belongs to the interval $\left[\widetilde{x}_{i}-\Delta_{i}, \widetilde{x}_{i}+\Delta_{i}\right]$. For example, if the measured value of the current is $\widetilde{x}=1 \mathrm{~A}$, and the manufacturer guarantees the measurement error to be within $\pm 0.1 \mathrm{~A}$, then the actual value of $x$ can be any number from the interval $[0.9,1.1]$.

In this case, the problem of estimating the error of indirect measurement can be reformulated as follows:

- we know $n$ intervals $\mathbf{x}_{i}=\left[\widetilde{x}_{i}-\Delta_{i}, \widetilde{x}_{i}+\Delta_{i}\right]$, 
- we know an algorithm $f$ which transforms $n$ real numbers $x_{1}, \ldots, x_{n}$ into a real number $y$, and

- we want to compute the interval

$$
\mathbf{y}=f\left(\mathbf{x}_{1}, \ldots, \mathbf{x}_{n}\right)=\left\{f\left(x_{1}, \ldots, x_{n}\right) \mid x_{i} \in \mathbf{x}_{i}\right\}
$$

This problem is called the basic problem of interval computations, and methods for solving this problem are called interval mathematics ${ }^{2,3,8}$.

\subsection{Linearization Is Not Always Possible}

If a function $f$ is smooth, and the errors $\Delta x_{i}$ are small, then we can neglect quadratic terms in $f$, and get explicit formulas for $\mathbf{y}$. Due to our approximation, we get approximate endpoints of the interval $\mathbf{y}$ : the actual values $y$ can be, therefore, slightly outside this approximate interval.

In many applications, it is OK, but in some real-life situations, the consequences of a possible error are so serious that we need to guarantee that $y$ is contained in the resulting interval $\mathbf{y}$.

\subsection{Interval Computations are Difficult}

In general, the interval computation problem is NP-hard even for quadratic functions $f\left(x_{1}, \ldots, x_{n}\right)$ (see, e.g., ${ }^{4}$ ). In plain English, this means that it is highly unprovable that we will be able to find a general feasible algorithm that computes the exact range for all functions $f$ and all intervals $\mathbf{x}_{i}$ in reasonable time. Since we cannot compute the exact range, what can we do instead?

We wanted to compute the exact range $\mathbf{y}$ because we wanted to get an interval that is guaranteed to contain the desired value $y$, and the range definitely contains this value. If we cannot compute the exact range in reasonable time, we can compute the approximate interval $\mathbf{Y}$ for the range. The only way to guarantee that the new interval still contains $y$ is to make sure that this new intervals contains the entire range $\mathbf{y} \subseteq \mathbf{Y}$, i.e., that this interval is an enclosure for the desired range.

In these terms, interval mathematics is an art of computing good narrow enclosures for the range of a given function $f\left(x_{1}, \ldots, x_{n}\right)$ on given intervals $\mathbf{x}_{1}, \ldots, \mathbf{x}_{n}$.

\subsection{Methods of Interval Mathematics: A Very Brief Introduction}

Interval mathematics started, in the 1950s, with the observation that for simple arithmetic operations $f\left(x_{1}, x_{2}\right)=x_{1}+x_{2}, x_{1}-x_{2}$, etc., the range can be computed explicitly; e.g.:

$$
\begin{gathered}
{\left[x_{1}^{-}, x_{1}^{+}\right]+\left[x_{2}^{-}, x_{2}^{+}\right]=\left[x_{1}^{-}+x_{2}^{-}, x_{1}^{+}+x_{2}^{+}\right]} \\
{\left[x_{1}^{-}, x_{1}^{+}\right]-\left[x_{2}^{-}, x_{2}^{+}\right]=\left[x_{1}^{-}-x_{2}^{+}, x_{1}^{+}-x_{2}^{-}\right]} \\
{\left[x_{1}^{-}, x_{1}^{+}\right] \cdot\left[x_{2}^{-}, x_{2}^{+}\right]=\left[\min \left(x_{1}^{-} \cdot x_{2}^{-}, x_{1}^{-} \cdot x_{2}^{+}, x_{1}^{+} \cdot x_{2}^{-}, x_{1}^{+} \cdot x_{2}^{+}\right),\right.} \\
\left.\max \left(x_{1}^{-} \cdot x_{2}^{-}, x_{1}^{-} \cdot x_{2}^{+}, x_{1}^{+} \cdot x_{2}^{-}, x_{1}^{+} \cdot x_{2}^{+}\right)\right] .
\end{gathered}
$$


The corresponding expressions are called formulas of interval arithmetic.

It turns out that we can use these expressions to get reasonable enclosures for arbitrary functions $f$. Indeed, when the computer computes the function $f$, it parses the function, i.e., it represents the computation as a sequence of elementary arithmetic operations. It can proven, by induction, that if we start with intervals and replace each arithmetic operation with the corresponding operation of interval arithmetic, at the end, we get an enclosure for $f$. For example, if $f(x)=x \cdot(1-x)$, represent $f$ as a sequence of two elementary operations:

- $r:=1-x(r$ denotes the 1 st intermediate result);

- $y:=x \cdot r$.

In the interval version, perform the following computations:

- $\mathbf{r}:=1-\mathbf{x}$

$\bullet \mathbf{y}:=\mathbf{x} \cdot \mathbf{r}$

In particular, when $\mathbf{x}=[0,1]$, compute the intervals $\mathbf{r}:=[1,1]-[0,1]=[0,1]$, and

$$
\mathbf{y}:=[0,1] \cdot[0,1]=[\min (0 \cdot 0,0 \cdot 1,1 \cdot 0,1 \cdot 1), \max (0 \cdot 0,0 \cdot 1,1 \cdot 0,1 \cdot 1)]=[0,1] .
$$

The interval $[0,1]$ is indeed an enclosure of the actual range $[0,0.25]$.

The enclosure obtained by using the above simple idea is often too wide. One of the main objectives of interval computations is to make this enclosure narrower. One way to do that is to use the mean value theorem, according to which $f(x)=$ $f\left(x_{0}\right)+f^{\prime}(\xi) \cdot\left(x-x_{0}\right)$ for some value $\xi$ between $x_{0}$ and $x$. Thus, if we take, as $x_{0}$, the midpoint of the interval $\mathbf{x}$ of width $w$, we will have $\left|x-x_{0}\right| \leq w / 2, f^{\prime}(\xi) \in f^{\prime}(\mathbf{x})$, and thus, $f(\mathbf{x}) \subseteq f\left(x_{0}\right)+f^{\prime}(\mathbf{x}) \cdot[-w / 2, w / 2]$. If we do not know the exact range $f^{\prime}(\mathbf{x})$, we can use the enclosure for this range. Similar formulas can be easily written for the case of several variables.

In many cases, the above idea leads to a reasonable enclosure. If the enclosure is still too wide, we can divide the original box $\mathbf{x}_{1} \times \ldots \times \mathbf{x}_{n}$ into sub-boxes, compute the enclosure for each of these subboxes, and then take the union of the resulting enclosures.

\section{Why Intervals in Aerospace Applications?}

Interval computations started with planning a mission to the Moon. To get guaranteed estimates for this problem, Ramon E. Moore, then Stanford's Ph.D. student working on 1959 NASA-oriented project, pioneered the new techniques.

Why methods of interval computations are needed in aerospace applications:

- First, we want to guarantee a mission, we want to guarantee that a spaceship hits the Moon (or another planet), and interval computations provide us with the guaranteed computation results. 
- Second, many NASA missions are missions into the unknown. We simply do not know the exact values of the parameters characterizing the distant planet's surface, or the corresponding probabilities; the only thing we may know for planning a mission are intervals of possible values of these parameters.

- Finally, one of the main goals of NASA missions is to produce solid scientific results, and "solid" means guaranteed.

In this paper, we will consider two case studies of using intervals in aerospace applications: processing remote sensing data and detecting possible faults in aerospace structures.

\section{Case Study: Reliable Sub-Division of Geological Areas}

This case study is described, in detail, in our paper ${ }^{1}$.

In geophysics, appropriate subdivision of an area into segments is extremely important, because it enables us to extrapolate the results obtained in some locations within the segment (where extensive research was done) to other locations within the same segment, and thus, get a good understanding of the locations which weren't that thoroughly analyzed. The subdivision of a geological zone into segments is often a controversial issue, with different evidence and different experts' intuition supporting different subdivisions.

For example, in our area - Rio Grande rift zone - there is some geochemical evidence that this zone is divided into three segments ${ }^{5}$ :

- the southern segment which is located, approximately, between the latitudes $y=29^{\circ}$ and $y=34^{\circ}$;

- the central segment - from $y=34.5^{\circ}$ to $y=38^{\circ}$; and

- the northern segment - from $y=38^{\circ}$ to $y=41^{\circ}$.

However, in the viewpoint of many researchers, this evidence is not yet sufficiently convincing.

It is therefore desirable to develop new techniques for zone sub-division, techniques which would be in the least possible way dependent on the (subjective) expert opinion and would, thus, be maximally reliable. To make this conclusion more reliable, we use, instead of the more rare geological samples, a more abundant topographical information (this information, e.g., comes from satellite photos). We can characterize each part of the divided zone by its topography.

In topographical analysis, we face a new problem: of too much data, most of which is geophysically irrelevant. To eliminate some of this irrelevant data, we can use the Fourier transform; indeed, it is known that while (at least some) absolute values of the map (forming a so-called spectrum) are geophysically meaningful, the phases usually are random and can be therefore ignored. So, we should only use the spectrum. 
Table 1:

\begin{tabular}{|c|c|c|c|c|c|c|}
\hline$y_{i}$ & 29 & 30 & 31 & 32 & 33 & 34 \\
\hline$s_{i}$ & 0.28 & 0.24 & 0.21 & 0.16 & 0.20 & 0.29 \\
\hline \hline 35 & 36 & 37 & 38 & 39 & 40 & 41 \\
\hline 0.31 & 0.35 & 0.46 & 1.00 & 0.80 & 0.96 & 0.74 \\
\hline
\end{tabular}

Since we are interested only in the large-scale classification, it makes sense to only use the spectrum values corresponding to relatively large spatial wavelengths, i.e., wavelengths $L$ for which $L \geq L_{0}$ for some appropriate value $L_{0}$. In particular, for the sub-division of the Rio Grande rift, it makes sense to use only wavelengths of $L_{0}=1000 \mathrm{~km}$ or larger.

Also, for the Rio Grande Rift, we are interested in the classification of horizontal zones, so it makes sense to divide the Rio Grande Rift into $1^{\circ}$ zones $\left[y^{-}, y^{+}\right]$(with $y$ from $y^{-}=30$ to $y^{+}=31$, from $y^{-}=31$ to $y^{+}=32, \ldots$, from $y^{-}=40$ to $\left.y^{+}=41\right)$. For each of these zones, we take the topographic data, i.e., the height $h(x, y)$ described as a function of longitude $x$ and latitude $y$, compute the Fourier transform $H(\omega, y)$ with respect to $x$, combine all the spectral values which correspond to large wavelength (i.e., for which $\omega \leq 1 / L_{0}$ ), and compute the resulting spectral value

$$
S\left(y^{-}\right)=\int_{y=y^{-}}^{y^{+}} \int_{\omega=0}^{1 / L_{0}}|H(\omega, y)|^{2} \mathrm{~d} \omega \mathrm{d} y .
$$

Since we are interested in comparing the spectral values $S(y)$ corresponding to different latitudes $y$, so we are not interested in the absolute values of $S(y)$, only in relative values. Thus, to simplify the data, we can normalize them by, e.g., dividing each value $S\left(y^{-}\right)$by the largest $S_{\max }$ of these values. In particular, for the Rio Grande rift, the resulting values of $y^{-}=y_{1}, y_{2}, \ldots$ and $s_{i}=S\left(y_{i}\right) / S_{\max }$ are as follows:

Based only on these spectral values $s_{i}$, we will try to classify locations into several clusters ("segments").

From the geophysical viewpoint, the desired zones correspond to "monotonicity regions": in the first zone, the values $s_{i}$ are (approximately) decreasing, in the next zone, they are (approximately) increasing, etc. So, we must look for the monotonicity regions of the (unknown) function $s(y)$.

The problem is that the values $s_{i}$ are only approximately known, so we cannot simply compare the values to determine whether a function increases or decreases. The heights are measured pretty accurately, so the only errors in the values $s_{i}$ come from discretization. In other words, we would like to know the values of the function $s(y)=S(y) / S_{\max }$ for all $y$, but we only know the values $s_{1}=s\left(y_{1}\right), \ldots, s_{n}=s\left(y_{n}\right)$ of this function for the points $y_{1}, \ldots, y_{n}$. For each $y$ which is different from $y_{i}$, it 
is reasonable to estimate $s(y)$ as the value $s_{i}=s\left(y_{i}\right)$ at the point $y_{i}$ which is the closest to $y$ (and, ideally, which belongs to the same segment as $y_{i}$ ). For each point $y_{i}$, what is the largest possible error $\Delta_{i}$ of the corresponding approximation?

When $y>y_{i}$, the point $y_{i}$ is still the closest until we reach the midpoint $y_{\text {mid }}=$ $\left(y_{i}+y_{i+1}\right) / 2$ between $y_{i}$ and $y_{i+1}$. It is reasonable to assume that the largest possible approximation error $\left|s(y)-s_{i}\right|$ for such points is attained when the distance between $y$ and $y_{i}$ is the largest, i.e., when $y$ is this midpoint; in this case, the approximation error is equal to $\left|s\left(y_{\text {mid }}\right)-s_{i}\right|$.

If the points $y_{i}$ and $y_{i+1}$ belong to the same segment, then the dependence of $s(y)$ on $y$ should be reasonably smooth for $y \in\left[y_{i}, y_{i+1}\right]$. Therefore, on a narrow interval $\left[y_{i}, y_{i+1}\right]$, we can, with reasonable accuracy, ignore quadratic and higher terms in the expansion of $s\left(y_{i}+\Delta y\right)$ and thus, approximate $s(y)$ by a linear function. For a linear function $s(y)$, the difference $s\left(y_{\text {mid }}\right)-s\left(y_{i}\right)$ is equal to the half of the difference $s\left(y_{i+1}\right)-s\left(y_{i}\right)=s_{i+1}-s_{i}$; thus, for $y>y_{i}$, the approximation error is bounded by $0.5 \cdot\left|s_{i+1}-s_{i}\right|$.

If the points $y_{i}$ and $y_{i+1}$ belong to different segments, then the dependence $s(y)$ should exhibit some non-smoothness, and it is reasonable to expect that the difference $\left|s_{i+1}-s_{i}\right|$ is much higher than the approximation error.

In both cases, the approximation error is bounded by $0.5 \cdot\left|s_{i+1}-s_{i}\right|$. Similarly, for $y<y_{i}$, the approximation error is bounded by $0.5 \cdot\left|s_{i}-s_{i-1}\right|$ if the points $y_{i}$ and $y_{i-1}$ belong to the same segment, and is much smaller if they don't. In both cases, the approximation error is bounded by $0.5 \cdot\left|s_{i}-s_{i-1}\right|$. We have two bounds on the approximation error and we can therefore conclude that the approximation error cannot exceed the smallest $\Delta_{i}$ of these two bounds, i.e., the value

$$
\Delta_{i}=0.5 \cdot \min \left(\left|s_{i}-s_{i_{1}}\right|,\left|s_{i+1}-s_{i}\right|\right) .
$$

As a result, instead of the exact values $s_{i}$, for each $i$, we get the interval $\mathbf{s}_{i}=\left[s_{i}^{-}, s_{i}^{+}\right]$ of possible values of $s(y)$, where $s_{i}^{-}=s_{i}-\Delta_{i}$ and $s_{i}^{+}=s_{i}+\Delta_{i}$. In particular, for the Rio Grande rift, we get:

$$
\begin{gathered}
\mathbf{s}_{1}=[0.26,0.30], \mathbf{s}_{2}=[0.225,0.255], \mathbf{s}_{3}=[0.195,0.225], \\
\mathbf{s}_{4}=[0.14,0.18], \mathbf{s}_{5}=[0.18,0.22], \mathbf{s}_{6}=[0.28,0.30], \\
\mathbf{s}_{7}=[0.30,0.32], \mathbf{s}_{8}=[0.33,0.37], \mathbf{s}_{9}=[0.405,0.515], \\
\mathbf{s}_{10}=[0.80,1.10], \mathbf{s}_{11}=[0.72,0.88], \mathbf{s}_{12}=[0.88,1.04], \\
\mathbf{s}_{13}=[0.63,0.85] .
\end{gathered}
$$

We want to find regions of uncertainty of a function $s(y)$, but we do not know the exact form of this function; all we know is that for every $i, s\left(y_{i}\right) \in \mathbf{s}_{i}$ for known intervals $\mathbf{s}_{i}$. How can we find the monotonicity regions in the situation with such interval uncertainty? Of course, since we only know the values of the function $s(y)$ in finitely many points $y_{i}$, this function can have as many monotonicity regions between $y_{i}$ and $y_{i+1}$ as possible. What we are interested in is funding the subdivision 
into monotonicity regions which can be deduced from the data. The first natural question is: can we explain the data by assuming that the dependence $s(y)$ is monotonic?

If not, then we can ask for the possibility of having a function $s(y)$ with exactly two monotonicity regions. If such a function is possible, then we are interested in possible locations of such regions. If such a function is not possible, then we will try to find a function $s(y)$ which is consisted with our interval data and which has three monotonicity regions, etc.

This problem was first formalized and solved $\mathrm{in}^{7}$, where we developed a lineartime algorithm for solving this problem. By applying this algorithm, we find three monotonicity regions: $[29,34],[31,41]$, and $[37,41]$ - in good accordance with the geochemical data from ${ }^{5}$.

\section{Case Study: Non-Destructive Testing}

This case study is described, in detail, in our papers ${ }^{6,9}$.

In many areas, e.g., in aerospace industry, in medicine, it is desirable to detect mechanical faults without damaging or reassembling the original system. For testing, we send a signal and measure the resulting signal. The input signal can be described by its intensity $r_{1}, \ldots, r_{n}$ at different moments of time. The intensities $s_{1}, \ldots, s_{m}$ of the resulting signal depend on $r_{i}: s_{j}=f_{j}\left(r_{1}, \ldots, r_{n}\right)$, where the functions $f_{j}$ depend on the tested structure.

Usually, we do not know the exact analytical expression for the dependency $f_{j}$, so we can use the fact that an arbitrary continuous function can be approximated by a polynomial (of a sufficiently large order). Thus, we can take a structure, try a general linear dependency first, then, if necessary, general quadratic, etc., until we find the dependency that fits the desired data.

If a structure has no faults, then the surface is usually smooth. As a result, the dependency $f_{j}$ is also smooth; we can expand it in Taylor series. Since we are sending relatively weak signals $r_{i}$ (strong signals can damage the plane), we can neglect quadratic terms and only consider linear terms in these series; thus, the dependency will be linear.

A fault is, usually, a violation of smoothness (e.g., a crack). Thus, if there is a fault, the structure stops being smooth; hence, the function $f_{j}$ stops being smooth, and therefore, linear terms are no longer sufficient. Thus, in the absence of fault, the dependence is linear, but with the faults, the dependence is non-linear. So, we can detect the fault by checking whether the dependency between $s_{j}$ and $r_{i}$ is linear. So, we send several different inputs, measure the values $r_{i}^{(k)}$ and $s_{j}^{(k)}$ corresponding to these inputs, and check whether the dependence is linear. In this case, the values $r_{i}^{(k)}$ and $s_{j}^{(k)}$ are the inputs $x_{1}, \ldots, x_{n}$, but the desired $q$ is a qualitative (yes-no) variable: we simply want to know whether there is a fault or not. If there is a fault, then we would also like to make a quantitative conclusion of its size, location, etc., but the most important part of the analysis is to check whether there is any fault at all. 
If the measurements were ideal, all we had to do was to check whether there are values $a_{j i}$ for which, for all $j$ and for all measurements $k$, we have:

$$
a_{j 0}+a_{j 1} \cdot r_{1}^{(k)}+\ldots+a_{j n} \cdot r_{n}^{(k)}=s_{j}^{(k)} .
$$

Solvability of a system of linear equations is easy to check.

In reality, the situation is more complicated. Measurement are usually imprecise: the result $\widetilde{x}$ of measuring the actual value $x$ is somewhat different from the actual value $x$. In many real-life situations, we do not know the probabilities of different values of measurement error $\Delta x=\widetilde{x}-x$, we only know the upper bound $\Delta$ of the corresponding measurement error. As a result, the only information that we have about the actual value $x$ of the measured quantity is that it belongs to the interval $\mathbf{x}=[x-\Delta, x+\Delta]$. So, in practice, instead of the exact values of $r_{i}^{(k)}$ and $s_{j}^{(k)}$, we have intervals $\mathbf{r}_{i}^{(k)}$ and $\mathbf{s}_{j}^{(k)}$ of possible values of these quantities. The question becomes: are these intervals consistent with the linearity, i.e., are there values $r_{i}^{(k)} \in \mathbf{r}_{i}^{(k)}$ and $s_{j}^{(k)} \in \mathbf{s}_{j}^{(k)}$ for which, for some values $a_{j i}$, the above linearity formulas hold.

In general, the solvability of the corresponding system of interval linear equations is an NP-hard problem ${ }^{4}$, but for some cases, efficient algorithms have been developed. For example, when we have only one (non-negative) input and only one output, with non-intersecting intervals $\mathbf{r}^{(1)}<\mathbf{r}^{(2)}<\ldots$, the solvability of the corresponding system of linear equations can be proven to be equivalent to the following inequality:

$$
\max _{k<l} \frac{s^{(l)-}-s^{(k)+}}{r^{(l)+}-r^{(k)-}} \leq \min _{k<l} \frac{s^{(l)+}-s^{(k)-}}{r^{(l)-}-r^{(k)+}} .
$$

We tested this method on the dependence of the energy $E$ of the ultrasound response on the voltage $V$ that causes the original ultrasound signal. The results show that non-linearity is indeed an indication of a fault:

For faultless plates, the above inequality is indeed true, meaning that the measurement results are consistent with linearity.

For plates with faults, this inequality is not satisfied, meaning that the dependence is non-linear.

\section{Other Applications and Future Work}

Other successful aerospace applications of interval techniques ${ }^{6}$ range from telemanipulation to robot navigation to analysis of multi-spectral satellite images.

Another application is to eliminating outliers from a gravity database. The University of Texas at El Paso hosts a huge complied database of gravity measurements. These measurements can be very useful for geophysics. The main problem with this database is that some measurement results are erroneous (outliers), and these erroneous results spoil the analysis. A natural approach to eliminating the outliers is as follows: if the difference between the measured gravitational forces in two nearby points is larger than physically possible, this means that one of these 
measurement results in an error. Since differences are small, at first glance, we can use linearization: $\Delta f \approx \sum \frac{\partial f}{\partial x_{i}} \cdot \Delta x_{i}$. However, in this case, linearization does not work because for the expression describing dependence of the gravitation field on the parameters derivatives can take arbitrary large values. Here, interval techniques help.

How can we make these applications even more successful? A natural way to do that is to take into consideration that engineers and scientists traditionally use statistical methods to describe uncertainty. In many practical situations, we do not know the probabilities, so intervals are a reasonable approach. In many practical cases, however, we have partial information about probabilities. Interval methods ignore this information; so, we need combined methods which would take this partial information into consideration.

\section{Acknowledgments}

This work was supported in part by NASA grants NCC5-209 and NCC 21232, by NSF grants DUE-9750858, CDA-9522207, ERA-0112968 and 9710940 Mexico/Conacyt, by the United Space Alliance grant NAS 9-20000 (PWO C0C67713A6), by the Air Force Office of Scientific Research grants F49620-95-10518 and F49620-00-1-0365, and by the National Security Agency grant MDA90498-1-0561.

\section{References}

1. D. D. Coblentz et al., "Towards Reliable Sub-Division of Geological Areas: Interval Approach", In: L. Reznik and V. Kreinovich (eds.), Soft Computing in Measurements and Information Acquisition, (Springer-Verlag, 2001, to appear).

2. R. B. Kearfott, Rigorous Global Search: Continuous Problems (Kluwer, Dordrecht, 1996).

3. R. B. Kearfott and V. Kreinovich (eds.), Applications of Interval Computations (Kluwer, Dordrecht, 1996).

4. V. Kreinovich et al., Computational complexity and feasibility of data processing and interval computations (Kluwer, Dordrecht, 1998).

5. N. J. McMillan, "Temporal and spatial magmatic evolution of the Rio Grande rift", New Mexico Geological Survey Guidebook, 49th Field Conference, Las Cruces County, 107-116, 1998.

6. S. A. Starks and V. Kreinovich, "Aerospace applications of soft computing and interval computations (with an emphasis on multi-spectral satellite imaging)", In: M. Jamshidi, M. Fathi, and T. Furunashi (eds.), Soft Computing, Multimedia, and Image Processing. Proceedings of the 2000 World Automation Congress WAC'2000, Maui, Hawaii, June 11-16, 2000 (TSI Press, Albuquerque, 2000) 644-651.

7. K. Villaverde and V. Kreinovich, "A linear-time algorithm that locates local extrema of a function of one variable from interval measurement results," Interval Computations, No. 4 (1993) 176-194.

8. website on interval computations http://www.cs.utep.edu/interval-comp

9. K. Worden et al., "Interval Methods in Non-Destructive Testing of Material Structures", Reliable Computing 7 (2001) 341-352. 